\title{
Measurement of gamma-rays from the keV-neutron capture by ${ }^{139} \mathrm{La}$
}

\author{
M. Igashira ${ }^{\mathrm{a}}$, M. Saito, J. Nishiyama, T. Ohsaki ${ }^{\mathrm{b}}$, and T. Katabuchi \\ Research Laboratory for Nuclear Reactors, Tokyo Institute of Technology, Tokyo 152-8550, Japan
}

\begin{abstract}
The capture cross sections and capture $\gamma$-ray spectra of ${ }^{139} \mathrm{La}$ were measured in a neutron energy region from 10 to $100 \mathrm{keV}$ and at $550 \mathrm{keV}$. Pulsed $\mathrm{keV}$ neutrons were produced by the ${ }^{7} \mathrm{Li}(\mathrm{p}, \mathrm{n})^{7} \mathrm{Be}$ reaction with a pulsed proton beam from a Pelletron accelerator. Capture $\gamma$-rays were detected with a large anti-Compton $\mathrm{NaI}(\mathrm{Tl})$ spectrometer by means of a time-of-flight method. A pulse-height weighting technique was applied to the observed capture $\gamma$-ray pulse-height spectra to obtain capture yields. Using the standard capture cross sections of ${ }^{197} \mathrm{Au}$, the capture cross sections of ${ }^{139} \mathrm{La}$ were derived with uncertainties of about $5 \%$. The capture $\gamma$-ray spectra were obtained by unfolding the observed capture $\gamma$-ray pulse-height spectra. The present results were compared with previous measurements and evaluated values.
\end{abstract}

\section{Introduction}

Since the neutron magic nuclides have small keV-neutron capture cross sections, nuclides with $\mathrm{N}=50,82$, and 126 act as bottlenecks in the chain of neutron capture reactions in the s-process nucleosynthesis. Therefore, their accurate keVneutron capture cross sections are important for the study on the s-process nucleosynthesis.

On the other hand, neutron capture $\gamma$-ray spectra include important information on physical quantities such as $\gamma$-ray strength function. Therefore, simultaneous analysis of both cross sections and $\gamma$-ray spectra will provide important information on the $\mathrm{keV}$-neutron capture reaction mechanism.

From these viewpoints, we have measured the keV-neutron capture cross sections and capture $\gamma$-ray spectra of ${ }^{139} \mathrm{La}(\mathrm{N}=$ $82)$. In the present paper, we report on the experimental results.

\section{Experimental procedure}

The experimental procedure has been described in detail elsewhere [1], so it is summarized here.

The capture cross sections and capture $\gamma$-ray spectra of ${ }^{139} \mathrm{La}$ were measured in an incident neutron energy region from 10 to $100 \mathrm{keV}$ and at $550 \mathrm{keV}$, using the 3-MV Pelletron accelerator of the Research Laboratory for Nuclear Reactors at the Tokyo Institute of Technology. An experimental arrangement is shown in figure 1.

Pulsed neutrons were produced by the ${ }^{7} \mathrm{Li}(\mathrm{p}, \mathrm{n})^{7} \mathrm{Be}$ reaction with a pulsed proton beam (1.5 ns width, $4 \mathrm{MHz}$ repetition rate) from the Pelletron accelerator. The incident neutron energy spectrum on a capture sample was measured by means of a time-of-flight (TOF) method with a ${ }^{6} \mathrm{Li}$-glass scintillation detector. A $5.0-\mathrm{mm}$ diameter by $5.0-\mathrm{mm}{ }^{6} \mathrm{Li}$-glass detector located $30 \mathrm{~cm}$ from the neutron source was used for the measurement in the 10-100 keV region, and a 102.0-mm diameter by $6.4-\mathrm{mm}{ }^{6} \mathrm{Li}$-glass detector located $460 \mathrm{~cm}$ was used for that at $550 \mathrm{keV}$.

${ }^{a}$ Presenting author, e-mail: iga@nr.titech.ac.jp

${ }^{b}$ Present address: Intellectual Property Bank Corp., Tokyo, Japan



Fig. 1. Experimental arrangement in the $10-100 \mathrm{keV}$ measurement.

The capture sample was natural $\mathrm{La}_{2} \mathrm{O}_{3}$ powder (about $50 \mathrm{~g}$ ) which was contained in a graphite case with an inner diameter of $55 \mathrm{~mm}$ and an inner length of $14 \mathrm{~mm}$. A gold $(\mathrm{Au})$ disk with a diameter of $55 \mathrm{~mm}$ and a thickness of $1 \mathrm{~mm}$ was used as a standard sample. Each capture sample was located at an angle of 0 degree with respect to the proton beam direction. The distance between the neutron source and the sample was $12 \mathrm{~cm}$ for the measurement in the $10-100 \mathrm{keV}$ region and $20 \mathrm{~cm}$ for that at $550 \mathrm{keV}$.

The capture $\gamma$-rays emitted from the ${ }^{139} \mathrm{La}$ or ${ }^{197} \mathrm{Au}$ sample were measured with a large anti-Compton $\mathrm{NaI}(\mathrm{Tl})$ spectrometer by means of a TOF method. The main NaI(Tl) detector of the spectrometer had a diameter of $15.2 \mathrm{~cm}$ and a length of $30.5 \mathrm{~cm}$, and was centered in a hollow Compton-suppression $\mathrm{NaI}(\mathrm{Tl})$ detector with an outer diameter of $33.0 \mathrm{~cm}$ and a length of $35.6 \mathrm{~cm}$. The spectrometer was set in a heavy shield consisting of borated paraffin, borated polyethylene, $\mathrm{Cd},{ }^{6} \mathrm{LiH}$ and potassium free lead [2]. The capture $\gamma$-rays were observed at an angle of 125 degree with respect to the proton beam direction. The signals from the spectrometer were recorded in a personal computer as two-dimensional data of pulse-height (PH) and TOF. The measurements with the ${ }^{139} \mathrm{La}$ sample (La run), the ${ }^{197} \mathrm{Au}$ sample (Au run), and 


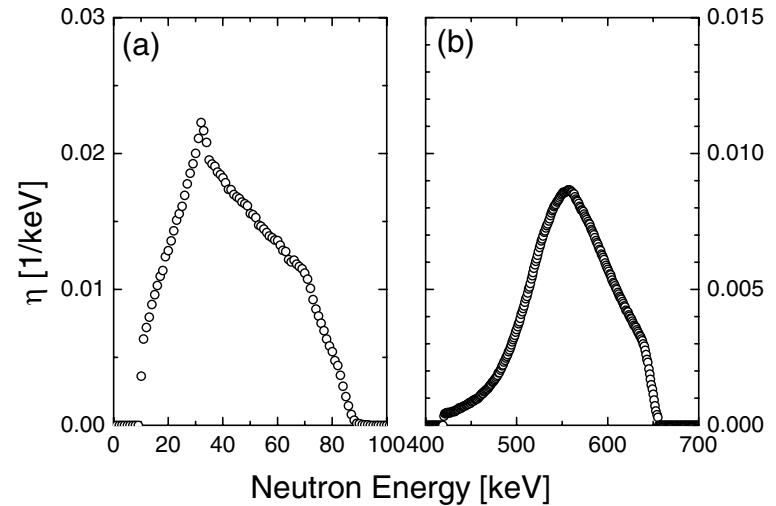

Fig. 2. Normalized energy spectra of incident neutrons in the (a) $10-100 \mathrm{keV}$ and (b) $550 \mathrm{keV}$ measurements.

no sample (Blank run) were performed cyclically to average out changes in experimental conditions such as the incident neutron spectrum.

\section{Data processing}

The data processing method has been also given in detail elsewhere [1], so it is summarized here.

The incident neutron energy spectrum on the sample in the $10-100 \mathrm{keV}$ or $550 \mathrm{keV}$ measurement was derived from the TOF spectrum observed with the small or large ${ }^{6} \mathrm{Li}$-glass detector in Blank run, respectively. The normalized spectra are shown in figure 2.

Figure 3 shows the TOF spectra observed with the $\gamma$-ray spectrometer in the 10-100 keV measurement. The broad peak below 550 channel in the spectrum for $\mathrm{La}$ run or $\mathrm{Au}$ run is due to the capture $\gamma$-rays from the corresponding sample, and the sharp peak around 650 channel is due to the $\gamma$-rays from the ${ }^{7} \mathrm{Li}(\mathrm{p}, \gamma){ }^{8} \mathrm{Be}$ reaction in the Li target. Four gates were set in the foreground region and one gate was set in the timeindependent background region, as shown in figure 3, and the corresponding PH spectra were obtained from the twodimensional data. Then, the net capture $\gamma$-ray $\mathrm{PH}$ spectra were obtained by subtracting the background $\mathrm{PH}$ spectra normalized with the gate-width ratios from the foreground $\mathrm{PH}$ spectra.

In order to obtain the capture yields corresponding to the individual gates of $\mathrm{La}$ and $\mathrm{Au}$ runs, a $\mathrm{PH}$ weighting technique [3] was applied to the net capture $\gamma$-ray PH spectra. The number of incident neutrons in Au run was determined by the capture yield of ${ }^{197} \mathrm{Au}$ and the averaged capture cross section of ${ }^{197} \mathrm{Au}$, which was obtained from the capture cross sections of ENDF/B-VI [4] and the normalized neutron energy spectrum. The number of incident neutrons in La run was derived from that in $\mathrm{Au}$ run and the neutron monitor counts of the ${ }^{6} \mathrm{Li}$-glass detector. The averaged neutron capture cross section of ${ }^{139} \mathrm{La}$ was derived from the number of incident neutrons and the capture yield of the ${ }^{139}$ La sample.

Corrections were made for the neutron self-shielding and multiple-scattering in the sample [5], for the $\gamma$-ray scattering and absorption in the sample, for the effect of the discrimination level $(600 \mathrm{keV})$ in the net capture $\gamma$-ray PH spectra on the

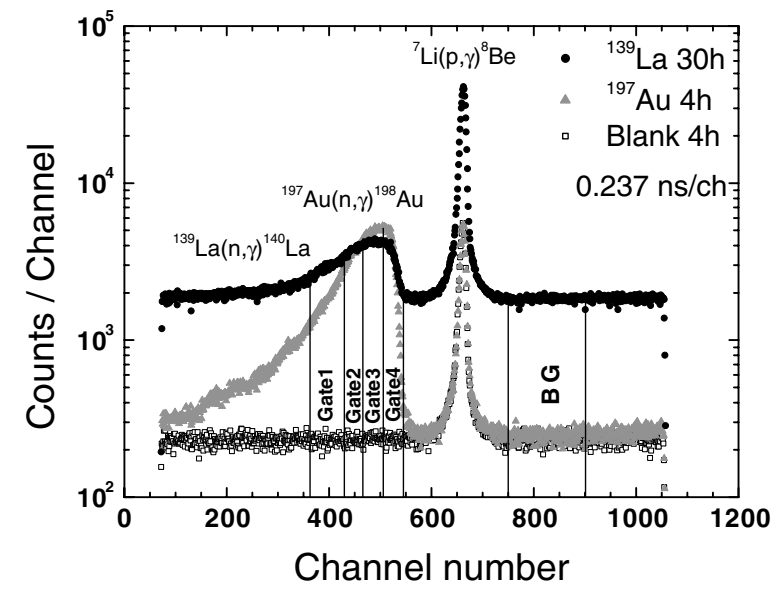

Fig. 3. TOF spectra observed with the $\gamma$-ray spectrometer in the 10-100 keV measurement.

capture yields, and for the effect of impurities in the sample on the capture yields.

The capture $\gamma$-ray spectra were derived by unfolding the net capture $\gamma$-ray PH spectra with a computer code, FERDOR [6], and the response matrix of the $\gamma$-ray spectrometer. The derived capture $\gamma$-ray spectra were normalized so that the integration of the spectrum with respect to the $\gamma$-ray energy is equal to the neutron binding energy plus the incident neutron energy [1].

\section{Results and discussion}

The capture cross sections of ${ }^{139} \mathrm{La}$ were derived with unceratinties of about $5 \%$ in the incident neutron energy region from 10 to $100 \mathrm{keV}$ and at $550 \mathrm{keV}$. The results in the 10-100 keV region are shown in figure 4, and compared with



Fig. 4. Neutron capture cross sections of ${ }^{139} \mathrm{La}$. 


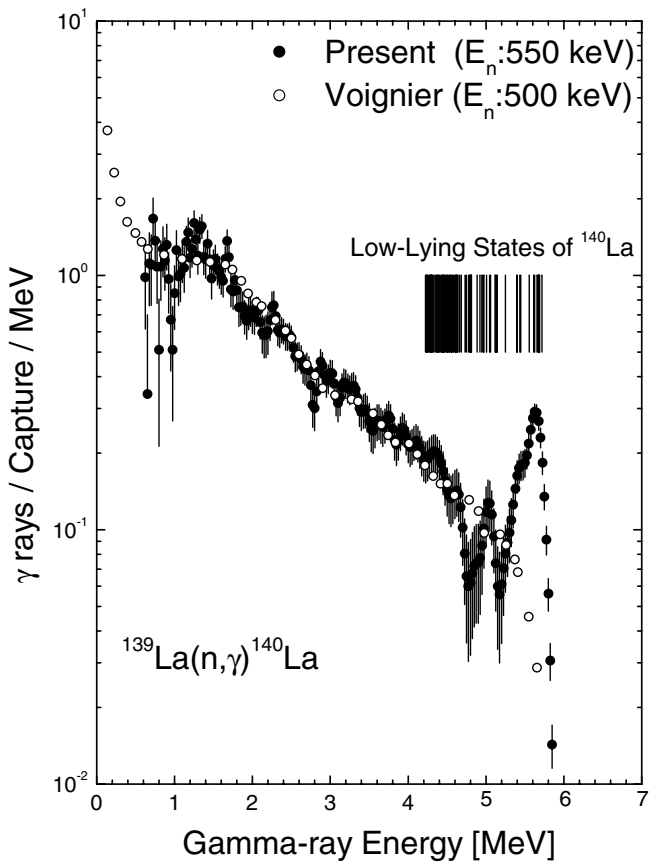

Fig. 5. Neutron capture $\gamma$-ray spectra of ${ }^{139} \mathrm{La}$.

previous measurements and the evaluations of JENDL-3.3 [7] and ENDF/B-VI [8]. Since activation methods are applicable to the measurement of capture cross sections of ${ }^{139} \mathrm{La}$, there are a considerable number of previous experimental results by activation methods. However, those results are not shown in figure 4.

The results of Musgrove et al. [9] are in good agreement with the present results. On the other hand, the result at $30 \mathrm{keV}$ of Macklin et al. [10] is larger than the present one by $25 \%$, and their result at $65 \mathrm{keV}$ is smaller than the present one by about $25 \%$. As for the results of Konks et al. [11], the neutron energy dependency of their results is different from that of the present results, although their result around $40 \mathrm{keV}$ is consistent with the present results.

The differences between both evaluations are less than $15 \%$ in the neutron energy region from 20 to $100 \mathrm{keV}$, and the presents results lie between both evaluations. It is worthy of note that the evaluations of ENDF/B-VII [12] in the region from 20 to $100 \mathrm{keV}$ are almost the same as those of JENDL-3.3.

The derived capture $\gamma$-ray spectrum of ${ }^{139} \mathrm{La}$ at $550 \mathrm{keV}$ is shown in figure 5, and compared with the result of Voignier et al. [13] at $500 \mathrm{keV}$. Low lying states of the residual nucleus, ${ }^{140} \mathrm{La}$, are shown in figure 5 . In the present spectrum, strong transitions from the neutron capture state to low lying states are clearly observed, although those transitions are not observed in the spectrum of Voignier et al. It is worthy of note that the data below $1.5 \mathrm{MeV}$ in the spectrum of Voignier et al. are not observed ones but calculated ones with a statistical model.

\section{Conclusion}

The neutron capture cross sections and capture $\gamma$-ray spectra of ${ }^{139} \mathrm{La}$ were measured in an energy region from 10 to $100 \mathrm{keV}$ and at $550 \mathrm{keV}$. The capture cross sections were derived with uncertainties of about $5 \%$. The derived cross sections in the region from 10 to $100 \mathrm{keV}$ lay between the JENDL-3.3 (and ENDF/B-VII) and ENDF/B-VI evaluations. The derived capture $\gamma$-ray spectra at $550 \mathrm{keV}$ was quite different from that of Voignier et al. at $500 \mathrm{keV}$ for the transitions from the neutron capture state to low-lying states of the residual nucleus.

\section{References}

1. S. Mizuno et al., J. Nucl. Sci. Technol. 36, 493 (1999).

2. M. Igashira, H. Kitazawa, N. Yamamuro, Nucl. Instrum. Meth. A 245, 432 (1986).

3. R.L. Macklin, J.H. Gibbons, Phys. Rev. 159, 1007 (1967).

4. ENDF/B-VI data file for ${ }^{197} \mathrm{Au}$ (MAT $\left.=7925\right)$, evaluated by P.G. Young (1991).

5. K. Senoo et al., Nucl. Instrum. Meth. A 339, 556 (1994).

6. H. Kendrick, S.M. Sperling, An Introduction to the Principles and Use of the FERDOR Code, Gulf Radiation Technology, GA9882 (1970).

7. JENDL-3.3 data file for ${ }^{139} \mathrm{La}(\mathrm{MAT}=5728)$, evaluated by $\mathrm{T}$. Aoki et al. (1993).

8. ENDF/B-VI data file for ${ }^{139} \mathrm{La}(\mathrm{MAT}=5728)$, evaluated by R.E. Schenter, F. Schmittroth (1980).

9. A.R. de Musgrove, B.J. Allen, R.L. Macklin, EXFOR 30384.004 (1991).

10. R.L. Macklin, N.H. Lazar, W.S. Lyon, Phys. Rev. 107, 504 (1957).

11. V.A. Konks, Yu.P. Popov, F.L. Shapiro, Soviet Phys. JETP 19, 59 (1964).

12. M.B. Chadwick, P. Oblozinsky et al. (CSEWG collaboration), ENDF/B-VII: Next Generation Evaluated Nuclear Data Library for Nuclear Science and Technology, Nucl. Data Sheets 107, 2931 (2006).

13. J. Voignier, S. Joly, G. Grenier, Nucl. Sci. Eng. 93, 43 (1986). 